\title{
Observation of the Internal Structure of Energetic Particle-Driven MHD Modes in a Large Helical Device
}

\author{
Tokihiko TOKUZAWA, Akira EJIRI ${ }^{1)}$, Kazuo KAWAHATA and LHD Experimental Group \\ National Institute for Fusion Science, Toki 509-5292, Japan \\ ${ }^{1)}$ Graduate School of Frontier Sciences, Tokyo University, Kashiwa 277-8561, Japan
}

(Received 15 November 2007 / Accepted 7 March 2008)

For the preparation study of a future burning plasma, experimental information about the internal structure of energetic particle-driven MHD instabilities, such as toroidal Alfvén eigenmodes, is important. In the Large Helical Device, three microwave reflectometer systems have been recently installed for measuring the density fluctuation in the wide radial region with a high spatial resolution. One of the three systems has three channels with fixed frequencies. The others are operated in a frequency-hopping mode. These systems are used for measuring the internal structures of an MHD mode and its temporal behaviors. Using these systems, strongly localized MHD instabilities driven by the energetic particles are measured in the plasma core region, and the measured values are consistent with the theoretical expectation.

(C) 2008 The Japan Society of Plasma Science and Nuclear Fusion Research

Keywords: Alfvén eigenmode, internal structure, MHD, density fluctuation, microwave reflectometer

DOI: $10.1585 /$ pfr.3.S1064

\section{Introduction}

In burning plasmas, energetic alpha particles enhance magnetohydrodynamics (MHD) modes such as toroidal Alfvén eigenmodes (TAEs). Also, the study of the energetic particle transport is one of the important issues, and it is found that the correlation between the temporal behavior of the instability and particle loss is quite high. In other words, the MHD mode affects the alpha-particle transport, and changes the plasma confinement. Therefore, energetic particle-driven MHD instability in several magnetic confinement devices has been studied [1-4]. In general, MHD phenomena can be observed using magnetic probes, and toroidal and poloidal mode numbers and traveling direction are obtained by an excellent analytical technique. However, in some large plasma experimental devices, the magnetic probe often failed to detect fluctuations that are located in the core region [1]. Theoretical analysis method using a three-dimensional code has been developed [5]. For the comparison between the simulation results and the results of the experiments, direct measurement of the internal radial distribution of these modes is important.

In the Large Helical Device (LHD [6]), we recently installed three types of microwave reflectometer systems for measuring the radial distribution of fluctuations. Microwave reflectometers have a potential of localized measurement by the cut-off effect in the plasma core region. The density perturbations $\delta n$ associated with the displacement $\boldsymbol{\xi}$ of a shear Alfvén mode is described in the equatorial plane by [2],

$$
\frac{\delta n}{n}=-\nabla \cdot \xi-\xi \cdot \frac{\nabla n}{n} \cong\left(\frac{-2 \hat{R}}{R^{2}}+\frac{\hat{n}}{L_{n}}\right) \cdot \boldsymbol{\xi}
$$

Here $n$ is the plasma density, $\hat{n}$ is the density unit vector normal to the magnetic surface, $R$ is the major radius, $\hat{R}$ is the unit vector along the major radius direction, and $L_{n}$ is the density scale length. Therefore, the reflectometers can be used to measure the internal structure of MHD phenomena, such as energetic particle mode and Alfvén eigenmodes.

At first, we used a fixed-frequency reflectometer system to measure the lower frequency range of the fluctuation such as an interchange mode, because of its limitation of the memory size of data acquisition. Recently, a real-time fast data acquisition system has been developed in LHD [7], and its sampling rate is up to $10 \mathrm{MSample} / \mathrm{sec}$. Using this system, high-frequency fluctuation measurement during the entire plasma discharge is possible. We also construct the frequency variable reflectometer systems, the so-called hopping reflectometer $[8,9]$. In order to measure the radial fluctuation profile in a wide range by reflectometry, many probing frequency components are required. However, this requires many microwave sources, which is economically not viable, and the obtained spatial resolution is limited. On the other hand, when the plasma condition is assumed to be steady during a frequency scanning period, the radial profile can be measured by the hopping system. In this paper, we describe these reflectometer systems, and present some experimental results. 


\section{Frequency Fixed $E$-band Reflec- tometer System}

$E$-band fixed-frequency heterodyne reflectometer system is used for the fluctuation measurement [10]. At present, the system has three channels with fixed frequencies of 78, 72, and $65 \mathrm{GHz}$. Combined three microwaves travel to/from the LHD through the corrugated waveguide for avoiding transmission loss. The microwaves launch from the outboard side along inverse major-radius direction on the equatorial plane. The polarization of the launching wave is set to the extraordinary mode, and the right-hand cut-off layer is used as the reflecting surface. The example of the cut-off position is shown in Fig. 1 (a). In this graph, the red solid line shows the radial profile
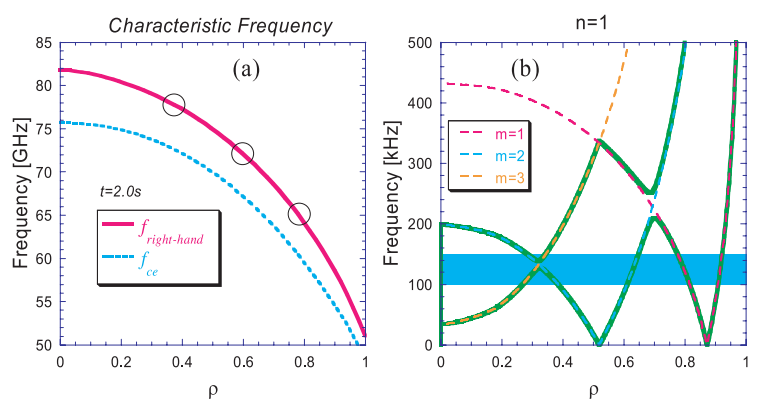

Fig. 1 (a) Radial profiles of right-hand cut-off frequency (red solid line) and electron cyclotron frequency (blue dotted line) (b) Radial profile of shear Alfvén spectra for $n=1$. of the right-hand cut-off frequency, and the corresponding cut-off layer with $78 \mathrm{GHz}$ is located around $\rho=0.35$. The simplified super-heterodyne detection technique is used for the receiver system. The signal is acquired using the realtime data acquisition system based on a compact PCI digitizer, and the sampling rate is usually $1 \mathrm{MHz}$. The system is quite convenient to observe MHD phenomena such as energetic particle driven Alfvén eigenmodes.

Examples of the temporal behavior of the reflectometer signal of $78 \mathrm{GHz}$, the magnetic probe signal, and the frequency power spectra are shown in Fig. 2. In this plasma discharge, bursts repeatedly occurred, and one of the bursts is shown in this figure. Coherent spectra of around 8 and $16 \mathrm{kHz}$ are caused by low- $n$ mode oscillation. In the range of $100-150 \mathrm{kHz}$, there are many other coherent modes. These modes are identified as the $n=1$ ( $n$ : toroidal mode number) TAE mode by the magnetic probe analysis. Figure 1(b) shows the radial profile of shear Alfvén spectra, and it is found that the frequency gap caused by the crossing of $m=2$ and $m=3$ branches is of around $130 \mathrm{kHz}$. On the reflectometer signal, higher frequency mode of around $230 \mathrm{kHz}$ is also observed. Just after $t=1.82 \mathrm{~s}$, an MHD-burst occurred, and TAE mode frequency components rapidly disappeared, as shown in Fig. 3. Then, $0.02 \mathrm{~s}$ later, this mode started to revive. This sudden disappearance may have been caused by the re-distribution of highenergy particles due to the MHD burst. In this discharge, the birth profile of energetic particles is determined by injecting the neutral beam, and its power is kept constant.
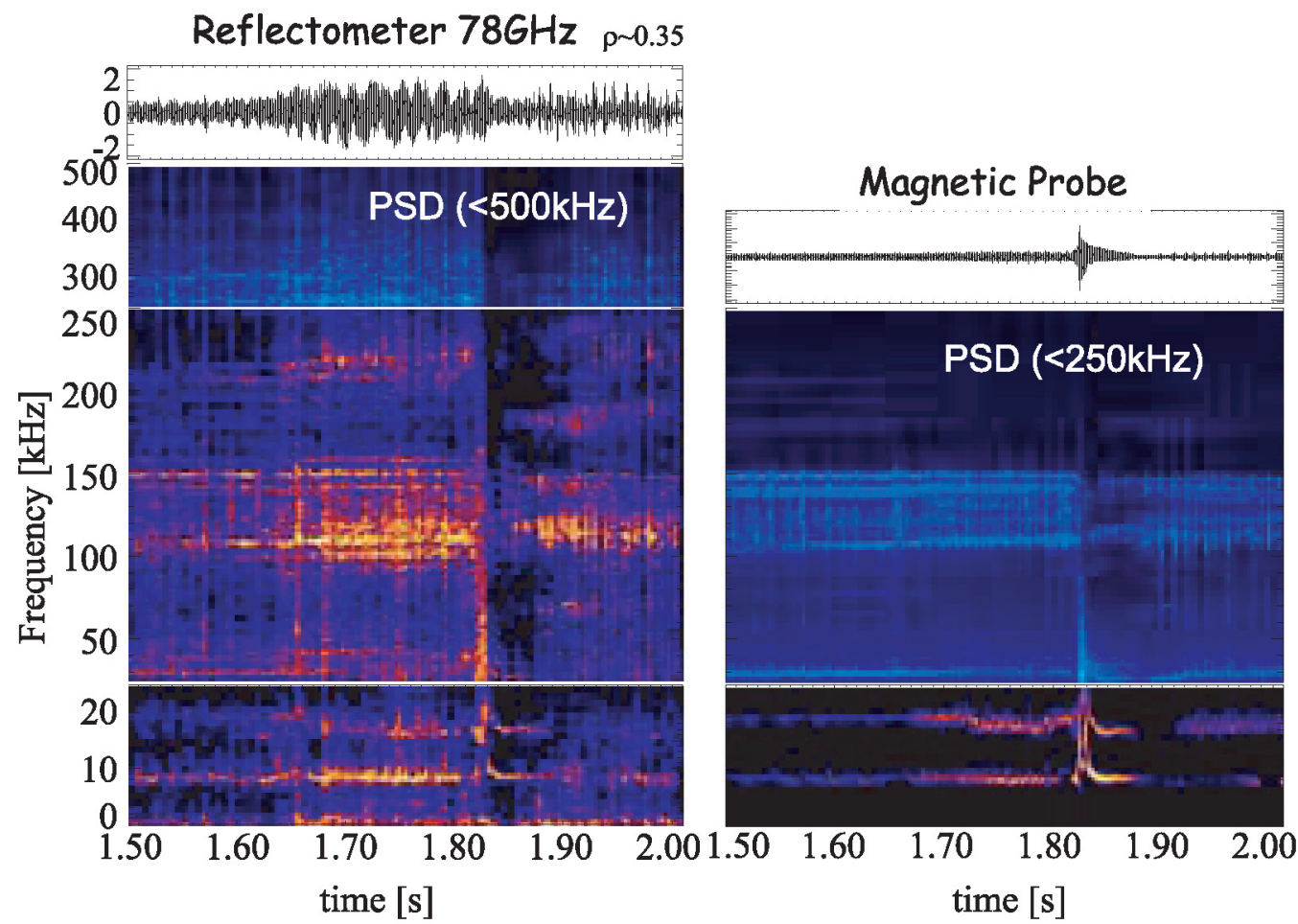

Fig. 2 Time evolution of reflectometer signal and frequency spectrum (Left) and those of magnetic probe signal (right). 
Therefore, the TAE mode is revived quickly, and it is maintained until the next burst. This system is suitable for fluctuation monitoring. Using this system, it is easy to obtain the following information: the time when the state of the fluctuation changes, the frequency at which the fluctuation

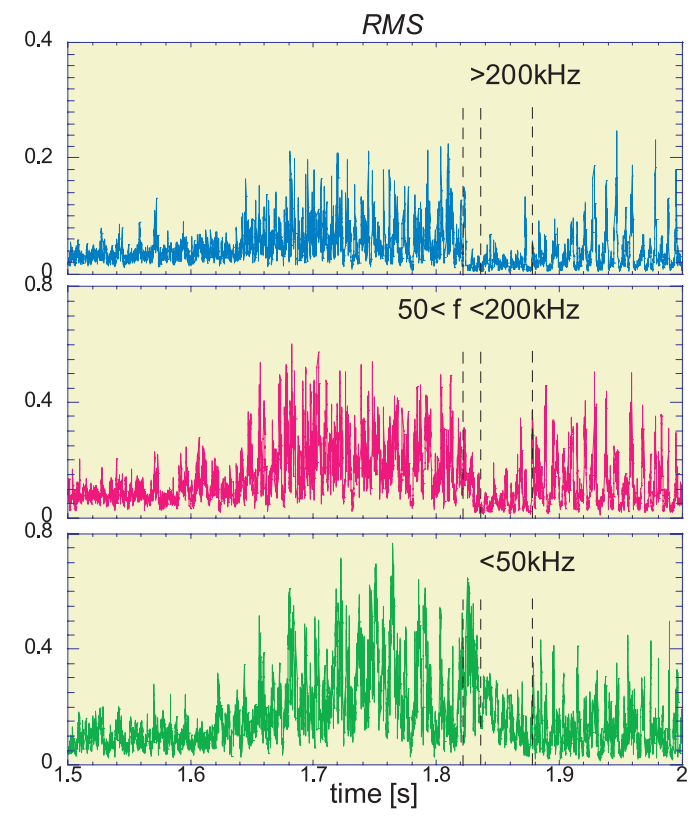

Fig. 3 Time evolution of fluctuation power of reflectometer signal. The frequency component higher than $200-\mathrm{kHz}$ (top), and that higher than $50-\mathrm{kHz}$ and less than $200-\mathrm{kHz}$ (middle), and that less than $50-\mathrm{kHz}$ (bottom), are shown. is observed in the plasma, etc. However, this system is a single horn system, and it is difficult to make it more precise.

\section{Frequency-Hopping $\mathrm{Ka}$-band Re- flectometer System}

There are two approaches for measuring the radial distribution of fluctuations using a reflectometer. One is using the multi-channel system, and another is using the wide-band frequency source system. For the latter system, source frequency is swept step-by-step in the entire frequency range. This type of frequency sweep is re-

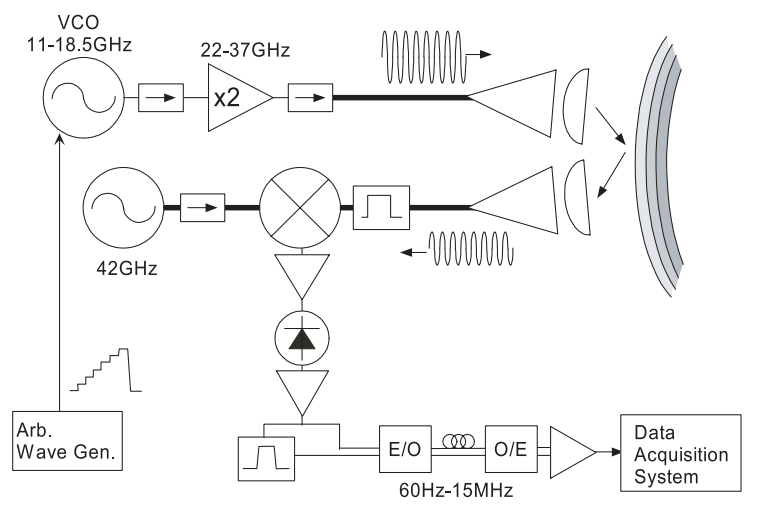

Fig. 4 Schematic view of Frequency Hopping $\mathrm{Ka}$-band Reflectometer system.

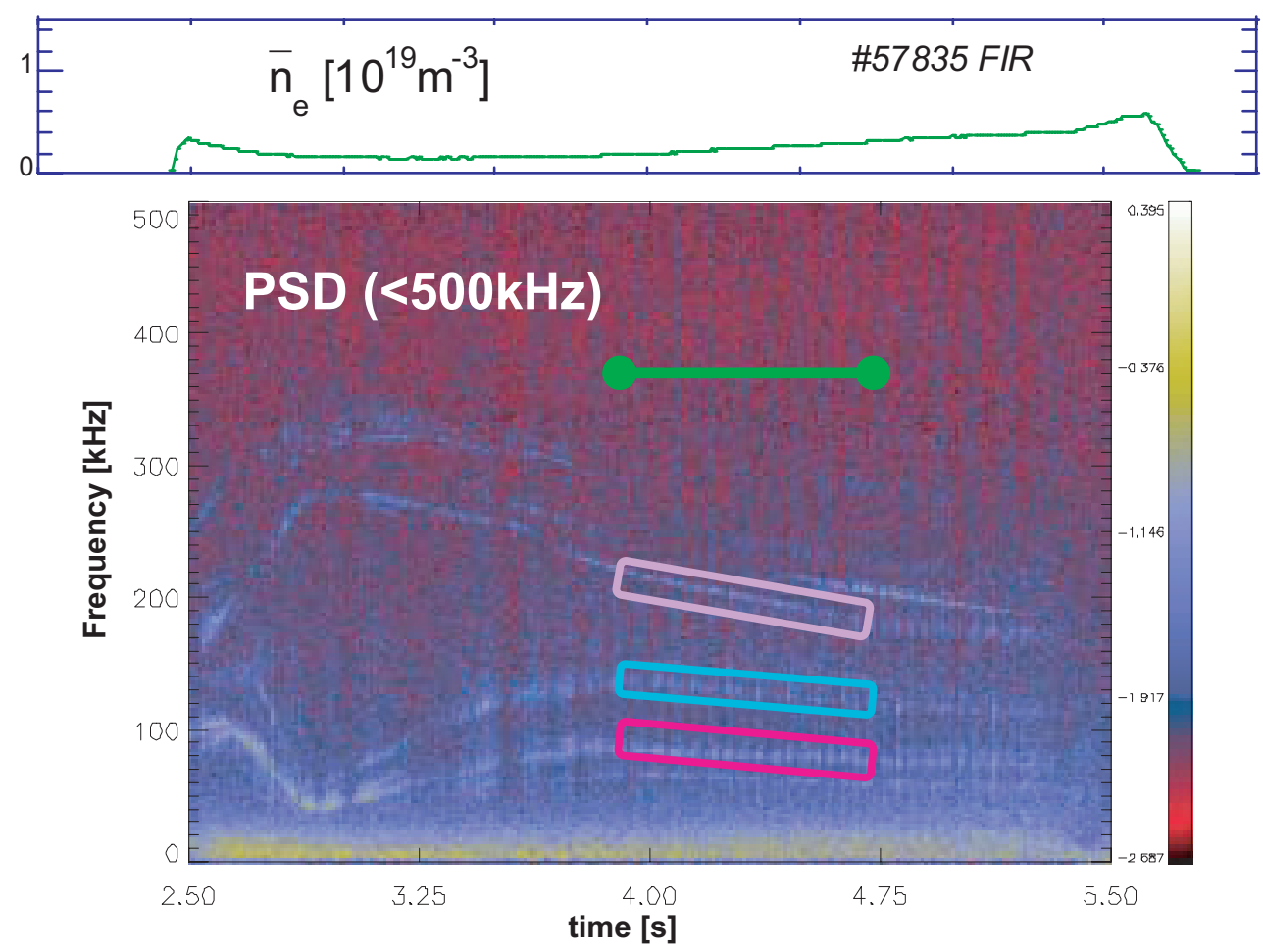

Fig. 5 Time evolution of electron density and frequency spectrum of interferometer mode CW Reflectometer. 
ferred to as frequency hopping. The step width is limited by the characteristic time of the measured fluctuation frequency. During the frequency change, the plasma condition and the fluctuation level are assumed to be constant. The schematic of frequency-hopping $\mathrm{Ka}$-band reflectometer system is shown in Fig. 4. The system uses a voltage controlled oscillator (VCO) as the source. The output frequency is easily changed by the external controlled signal. The wave is amplified, and this frequency is multiplied by two. The reflected wave is mixed with a local wave for heterodyne detection, and the intermediate frequency (IF) signal is amplified and detected. The data acquisition system used here is the same as the one used in the previous $E$-band reflectometer system.

Here, we show one example of radial distribution measurement. In the experiment, the axial magnetic field strength is $1.0 \mathrm{~T}$, the averaged electron density is under $0.5 \times 10^{19} \mathrm{~m}^{-3}$, as shown in Fig. 5, and the neutral beam is injected with constant power. The source frequency is swept in the entire frequency range every $200 \mathrm{~ms}$ and the number of the frequency step is 20 in this plasma shot. The time duration of one launching frequency is about $8 \mathrm{~ms}$, and the data sampling rate is $1 \mu \mathrm{s}$. Then the number of data points is 8,000 , and the frequency resolution is about $125 \mathrm{~Hz}$ for each frequency component. These parameters are appropriate for observing the MHD phenomena such as TAE. Figure 5 shows the frequency spectrum of the previous frequency fixed at $78-\mathrm{GHz}$ reflectometer signal. In this plasma condition, there is no corresponding cut-off layer of the $78-\mathrm{GHz}$ wave; then this system is operated as an interferometer mode. We can observe several continuous coherent frequency components. Figures $6(d)$ and (e) show the radial profiles of the fluctuation strength of the frequency hopping reflectometer signal at $t=4.0-4.8 \mathrm{~s}$ (4 sweep periods). It can be seen that the frequency component around $200 \mathrm{kHz}$ is large at $\rho=\sim 0.8$, and the other components around 150 and $80 \mathrm{kHz}$ are localized at the plasma center. Here, the data points, which are located at negative $\rho$, represent the case when these frequency waves are not reflected from the plasma, but they pass through the plasma and are reflected back from the opposite wall. The calculated shear Alfvén spectra are also shown in Fig. 6 (c). The frequency gap of around $200 \mathrm{kHz}$ is located at $\rho=\sim 0.7$. It shows good agreement with the measured profile data. On the other hand, the coherent frequency of around $80 \mathrm{kHz}$ is lower than the gap frequency, and the temporal behavior is different from the $200-\mathrm{kHz}$ frequency mode. One possibility of this fluctuation is an energetic particle mode (EPM). The frequency of $150 \mathrm{kHz}$ is located close to the other gap frequency, but the mode structure and temporal behavior are similar with the $80-\mathrm{kHz}$ component. Therefore, the identification of these modes is still to be verified. Also, strictly speaking, the density fluctuation is related with the phase difference of the reflected signal, and currently, the measured signal of this system is described by $A \cos \phi$. Here, $A$ is the amplitude and $\phi$ is the phase of the
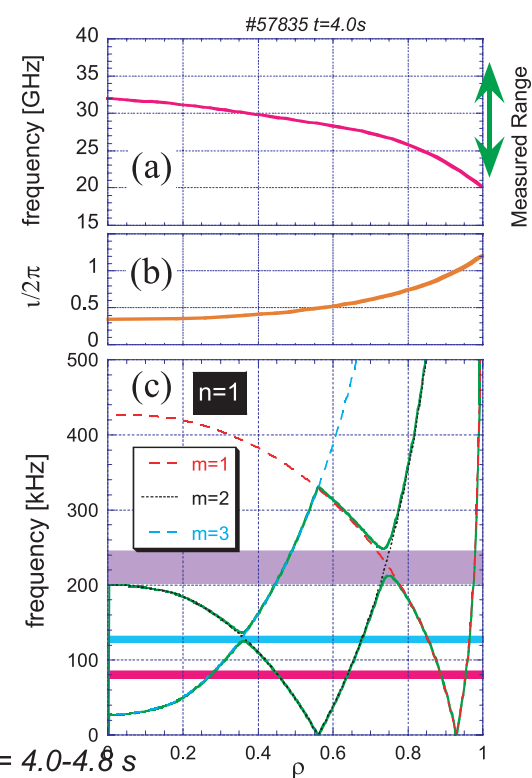

$\# 57835 t=4.0-4.8 \mathrm{~s}$
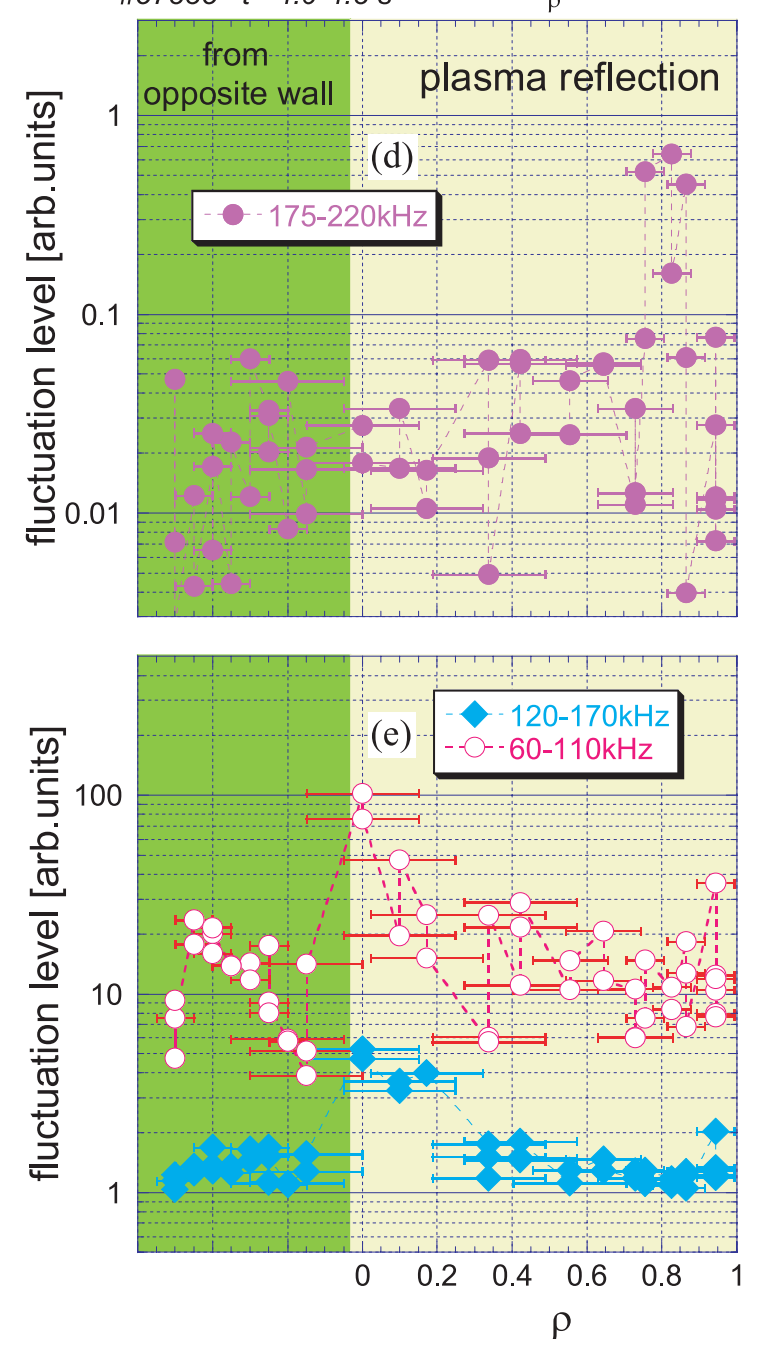

Fig. 6 Radial profiles of (a) right-hand cutoff frequency, (b) iota, and (c) Shear Alfven spectra for $n=1$. Radial profiles of fluctuation component, obtained by $\mathrm{Ka}$-band Hopping reflectometer, which is integrated in the frequency ranges of (d) $175-220 \mathrm{kHz}$, and (e) both $120-170 \mathrm{kHz}$ and $60-$ $110 \mathrm{kHz}$, respectively. 
detected signal, which is not directly related to the density fluctuation. Since this system was originally designed for density profile measurements, the system has not been optimized for fluctuation measurements. Therefore, the direct phase measurement of the reflected wave is necessary for an accurate analysis of the fluctuation.

\section{Frequency Hopping $V$-band Reflec- tometer System}

For more accurate fluctuation measurement, we have developed a new system shown in Fig. 7. Some components are added to the previous $\mathrm{Ka}$-band system. In particular, the single side-band (SSB) frequency modulation is utilized for the direct phase measurement. Also, a synthesizer is used as a source with a low phase-noise. The signal-to-noise ratio was measured to be $50 \mathrm{~dB}$.

A preliminary experimental result of this hopping system is obtained, and an example is shown in Fig. 8. In this figure, the launching frequency is changed from 52 to $63 \mathrm{GHz}$, and the step size is $1 \mathrm{GHz}$ with a $50-\mathrm{ms}$ duration. Some Alfvén eigenmodes can be observed in the entire launching frequency range. Unfortunately, the fluctuation level and its frequency are not constant in this discharge, and the mode structure of the fluctuation can not be obtained. We have planned to improve this system, and will obtain internal information of Alfvén eigenmodes in the near future.

\section{Summary}

To study the internal structure of Alfvén eigenmodes, three reflectometer systems are installed in a LHD. Energetic particle-driven mode such as TAE, can be observed with high-spatial and temporal resolutions. Using the frequency hopping technique, the internal structure can be obtained. For accurate analysis, the direct phase measurement system was used and preliminary signals were obtained. More improvements, such as a power amplifier of the probe beam and a narrower band pass filter, are planned for achieving a better signal-to-noise ratio, which will contribute to the study of Alfvén eigenmodes in the near future.

\section{Acknowledgements}

This study was partially supported by a Grant-in-Aid for Scientific Research from the Ministry of Education, Culture, Sports, Science and Technology (Nos. 18035015 and 18686075), and also by NIFS07ULHH507 from the budget grant-in-aid of the National Institute for Fusion Sci-

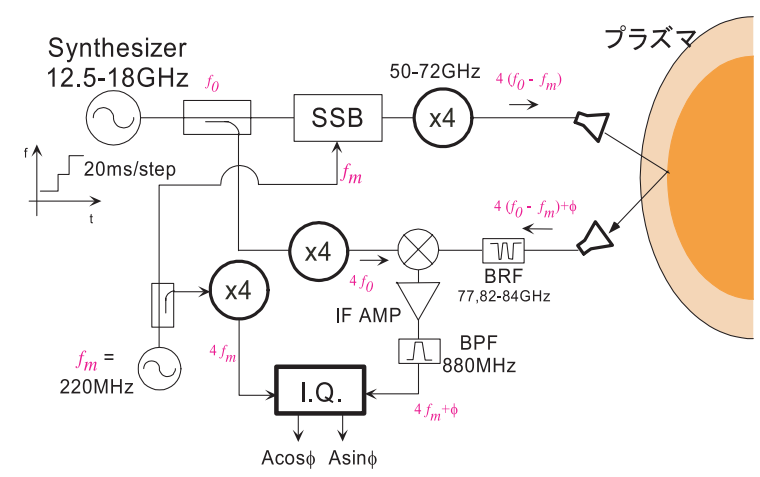

Fig. 7 Schematic view of Frequency Hopping $V$-band Reflectometer system.

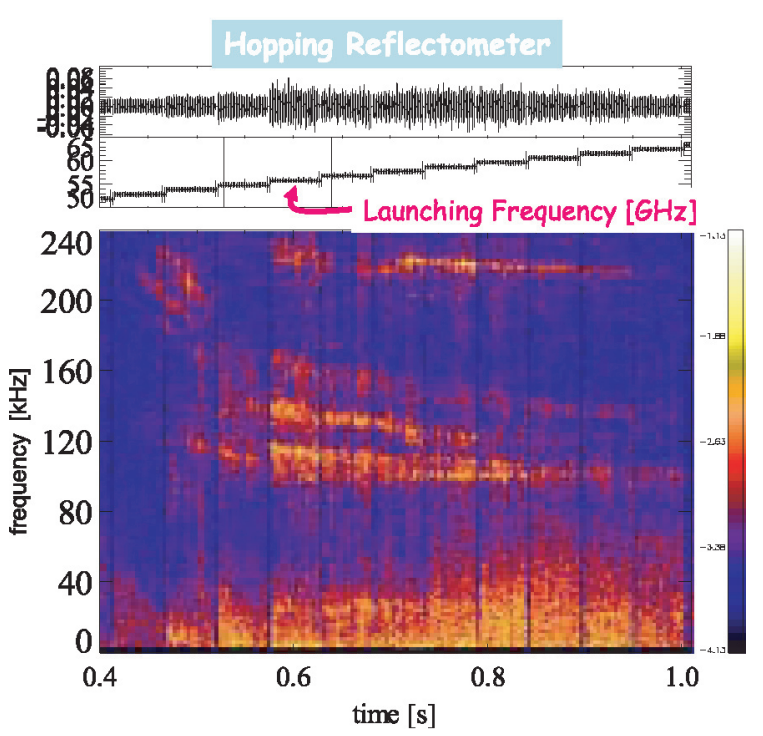

Fig. 8 Temporal behavior of the Frequency Hopping V-band Reflectometer signal and its frequency spectra.

ence.

[1] S.E. Sharapov et al., Phys. Rev. Lett. 93, 165001 (2004).

[2] R. Nazikian et al., Phys. Rev. Lett. 91, 125003 (2003).

[3] H. Kimura et al., Nucl. Fusion 38, 1303 (1998).

[4] S. Yamamoto et al., Nucl. Fusion 45, 326 (2005).

[5] C. Nührenberg, Phys. Plasmas 6, 137 (1999).

[6] O. Motojima et al., Nucl. Fusion 43, 1674 (2003).

[7] H. Nakanishi et al., Fusion Eng. Des. 66-68, 827(2003).

[8] L. Cupio et al., Rev. Sci. Instrum. 75, 3865 (2004).

[9] S. da Graca et al., Plasma Phys. Control. Fusion 49, 1849 (2007).

[10] T. Tokuzawa et al., 31st EPS Conf. P5-114 (2004). 